\title{
La viola de cocho du mato grosso*
}

\section{Luis Fernandez}

\section{Q OpenEdition \\ Journals}

Édition électronique

URL : http://journals.openedition.org/ethnomusicologie/2337

ISSN : 2235-7688

Éditeur

ADEM - Ateliers d'ethnomusicologie

Édition imprimée

Date de publication : 1 janvier 1989

Pagination : 151-164

ISBN : 2-8257-0178-5

ISSN : $1662-372 X$

Référence électronique

Luis Fernandez, "La viola de cocho du mato grosso* », Cahiers d'ethnomusicologie [En ligne], 2 | 1989, mis en ligne le 15 septembre 2011, consulté le 01 mai 2019. URL : http://journals.openedition.org/ ethnomusicologie/2337 


\title{
LA VIOLA DE COCHO DU MATO GROSSO ${ }^{1}$
}

\author{
Luis Fernandez
}

La viola de cocho (littéralement, «luth en forme d'auge») est l'instrument principal de trois danses populaires du Mato Grosso, plus précisément de la région de Cuiabà: le cururù, le siriri et la dança de São Gonçalo.

Cet article s'inspire, en partie, du seul travail approfondi existant sur le sujet, soit la thèse de Julieta de Andrade, Cocho Matogrossense: um alaùde brasileiro (1981). En effet, si l'instrument n'apparaît pratiquement pas dans la bibliographie spécialisée, c'est que les organologues n'ont montré qu'une curiosité limitée pour la forme étrange de l'instrument, pour sa sonorité inhabituelle et pour l'absence d'orifice acoustique qui semble empêcher la propagation des sons. Par ailleurs, l'instrument étant pratiqué dans les catégories moyennes et laborieuses de la population, il est très fréquent de trouver des personnes qui ont entendu parler de la viola de cocho, mais qui n'en ont jamais $\mathrm{vu}$, malgré sa large diffusion dans la région. Finalement, le caractère intime, humble et réservé de la relation entre le musicien et l'instrument a en quelque sorte préservé ce dernier en freinant sa propagation.

\section{La région et l'homme}

La viola de cocho est l'apanage des habitants de la Cuiabãnia, nom donné à l'aire géo-économique située aux environs de Cuiabà, capitale du Mato Grosso. Parmi ses municipios («communes»), les principaux sont Varzea Grande, Santo Antonio de Leverger, Nossa Senhora do Livramento, Poconé et Chapada dos Guimaraẽs.

A ne pas confondre avec la viola caipira à cordes métalliques, répandue dans tout le pays. Cocho signifie «auge», «mangeoire»; l'origine du nom est controversée. 
La population de cette région étendue est très inégalement répartie, sa densité atteignant pour tout l'État du Mato Grosso une moyenne de 1.4 habitant par $\mathrm{km}^{2}$. L'économie est basée sur les activités agro-pastorales (principalement l'élevage extensif des bovins et la riziculture). A cela s'ajoute aujourd'hui l'exploitation des ressources minières et l'implantation d'industries. A l'exception de Cuiabà, dont le centre est de plus en plus envahi par de grands édifices, les villes présentent un aspect colonial ancien avec une majorité de maisons en pisé, entourées de jardins ou de vergers. Même Cuiabà présente le même aspect dès que l'on quitte le centre, tant par les façades que par l'agencement intérieur des maisons.

Les différences sociales sont beaucoup plus marquées à Cuiabà qu'à l'intérieur de l'État. Dans la capitale résident les familles des propriétaires terriens, les commerçants venus des grands villes du Sud, quelques industriels et l'élite intellectuelle. Tout ce monde côtoie cordialement les classes laborieuses composées des fonctionnaires, des employés, du personnel du commerce et des familles immigrées venues de diverses régions ou États, qui constituent la majorité de la main-d'oeuvre non qualifiée.

Les violeiros ou cururueiros ${ }^{2}$, comme on les appelle, se recrutent dans la main-d'ouvre ouvrière, rarement dans la classe moyenne. Il s'agit d'aidesmaçons, de charpentiers, d'ouvriers de chantier, d'agriculteurs, de travailleurs de ferme ou de garimpo (exploitation de minerai). Le violeiro vit comme l'habitant pauvre de la région: il travaille toute la journée, et il se détend et s'amuse la nuit et en fin de semaine.

\section{L'instrument}

La viola de cocho est un cordophone aux cordes pincées et "grattées», qui s'apparente aux luths piriformes.

Deux sortes de bois sont employées de préférence pour la construction du corps: celui de la sarâ ou sarâ de leite ${ }^{3}$ (euphorbiacée), et celui de la ximbuva ou timbuva $a^{4}$ (légumineuse mimosacée). Plus rarement, on utilise la figueira ${ }^{5}$ (moracée).

A ne pas confondre avec la viola caipira à cordes métalliques, répandue dans tout le pays. Cocho signifie «auge», "mangeoire»; l'origine du nom est controversée.

2 Les joueurs de viola sont appleés violeiros, tandis que le terme de cururueiro se réfère plus particulièrement au membre d'un groupe dansant le cururù, qu'il joue de la viola de cocho ou du ganzà.

3 Synonyme de sarandi (du tupi) selon les informateurs; il s'agit d'un arbre poussant en abondance au bord des rivières.

4 Ximbuva, timbuva ou tamboril sont des noms populaires correspondant à timbaúba, du tupi timo'iua.

$5 \quad$ Il existe environ vingt-sept espèces du genre ficus au Brésil; les violas de figueira sont faites d'une rampa, c'est-à-dire d'une grande racine externe. 
Selon Manoel Severino, artisan renommé et ami personnel, «ce n'est pas tellement le choix du bois qui importe, mais l'artisan et la manière de faire».

La première opération consiste à prélever dans la forêt, au bord de l'eau ou ailleurs selon l'espèce, un grand parallélépipède de bois à l'aide d'une hache (la scie serait un affront pour les puristes!).

Une fois coupée dans le sens longitudinal des fibres, la pièce de bois est laissée à sécher quelques jours pour éviter d'éventuelles fentes ou déformations. Sur une des grandes faces est ensuite tracé au crayon le contour de la viola. Pour ce faire, on prend pour modèle une autre viola qui est alors appliquée directement sur le tronçon de bois. L'autorisation en est demandée au propriétaire de l'instrument que l'on voudrait copier, et elle est toujours obtenue, une telle requête équivalant en fait à un éloge. Une autre possibilité consiste à se servir d'un chablon de bois ou de carton. Cette manière de procéder explique pour une large part les différences de forme et de dimensions rencontrées d'une viola à l'autre.

Les contours de l'instrument sont façonnés à l'aide d'une hache et d'une machette. A ce stade, le tronçon de bois «est déjà une viola», d'après l'artisan (fig. 1).

Il s'agit ensuite de creuser la cavité de la caisse de résonance, puisque l'instrument est fait d'un seul bloc (fig. 2). C'est cette particularité qui a donné son nom de cocho («auge»). Le travail est commencé avec une herminette (fig. 3) et soigneusement fini avec divers ciseaux de menuisier (fig. 4-6), pour laisser une épaisseur, au niveau du fond et des éclisses, d'un centimètre environ. La sonorité dépendra de l'épaisseur de la caisse et de sa finition intérieure. « $\mathrm{Si}$ vous faites une de ces violas en laissant des traces de coupe à l'intérieur, sans les lisser au papier de verre, sans finition, elle ne peut jamais être bonne. Ces traces, ces entailles dans le bois à l'intérieur, vous savez? Ça empêche le son» (Manoel Severino).

Pour la fabrication de la table d'harmonie, on recherche des bois légers et flexibles, et on utilise de préférence la racine de la figueira. On découpe des plaques dans les parties externes des racines (certains artisans disent cependant que les parties enterrées vibrent mieux) qu'il faut ensuite laminer patiemment au couteau jusqu'à une épaisseur de deux millimètres environ; mais il arrive de plus en plus fréquemment que l'on fasse faire ce travail par le menuisier. La finition au papier de verre revient cependant toujours à l'artisan.

Le stade suivant consiste dans le collage de la table d'harmonie au moyen de la sève des tubercules de l'orchidacée sumaré ou sumbaré ${ }^{6}$. Ces petits tubercules, formant racine, sont coupés et raclés afin d'en recueillir le jus sur la pointe d'un couteau; ce jus est ensuite appliqué sur les bords de la viola et le pourtour de la table d'harmonie. Pour en faciliter le séchage, qui dure quelques heures, l'instrument est fermement entouré de cordelettes de coton. En cas d'urgence, le séchage peut être accéléré en utilisant du jus du sumaré chauffé

6 Cyrtopodium Paranaensis et Cyrtopodium Andersonii. L'une est terrestre, l'autre parasite. 


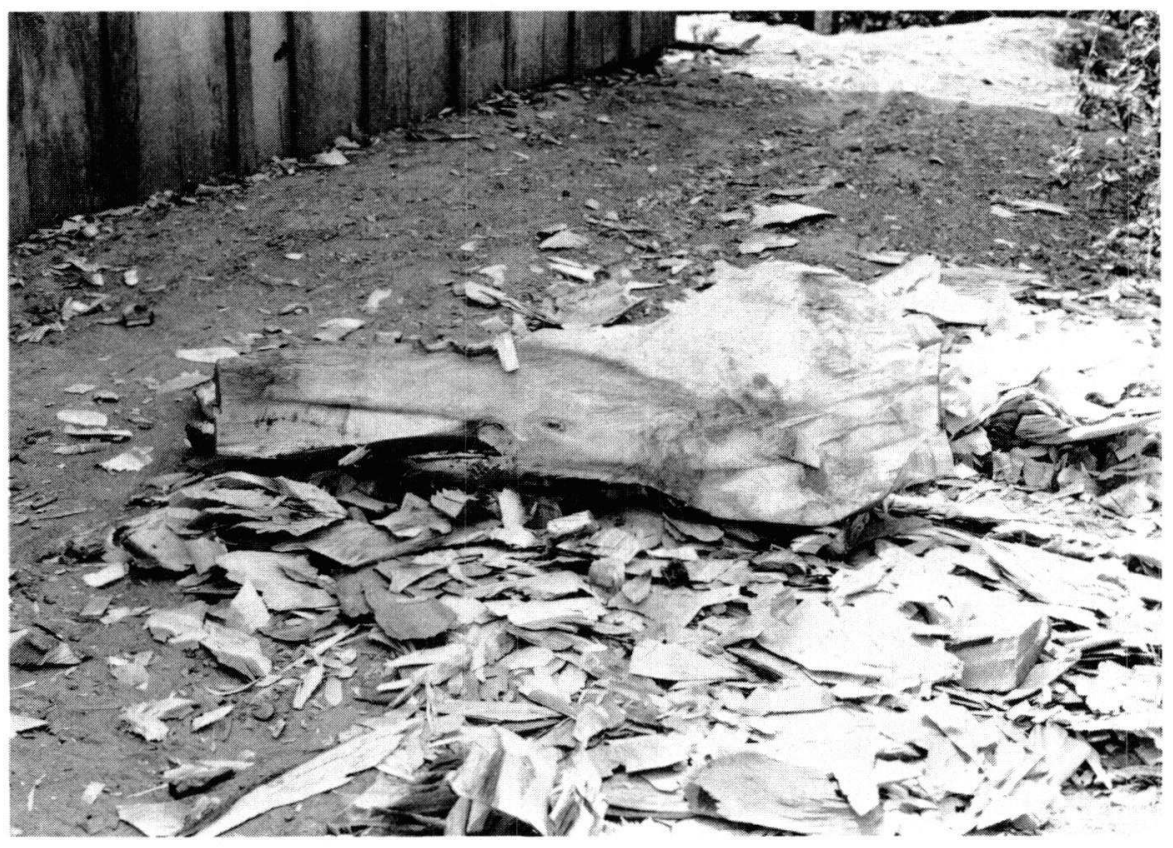

Fig. 1: «Le tronçon de bois grossièrement découpé s'appelle déjà une viola», selon les artisans. Photo: L. Fernandez, 1987.

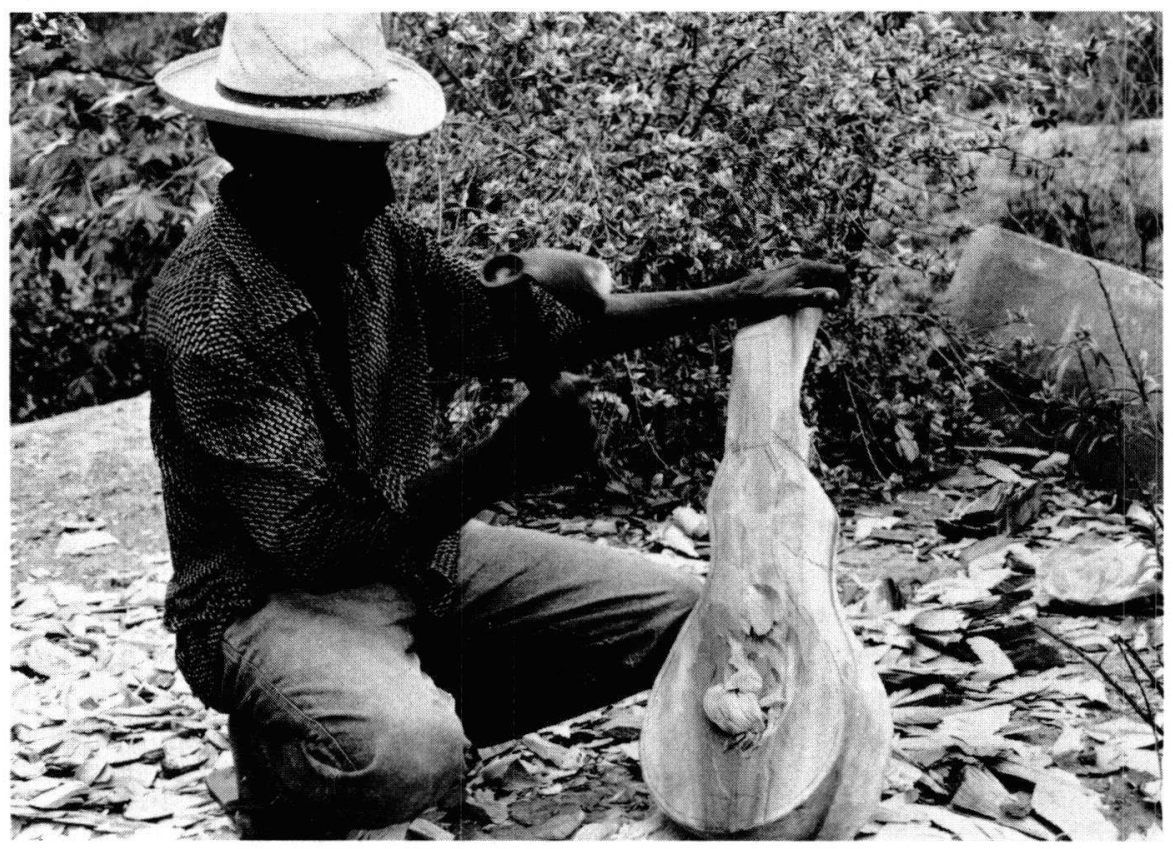

Fig. 2: Début de travail pour l'obtention de la cavité de la viola. Photo: L. Fernandez, 1987. 


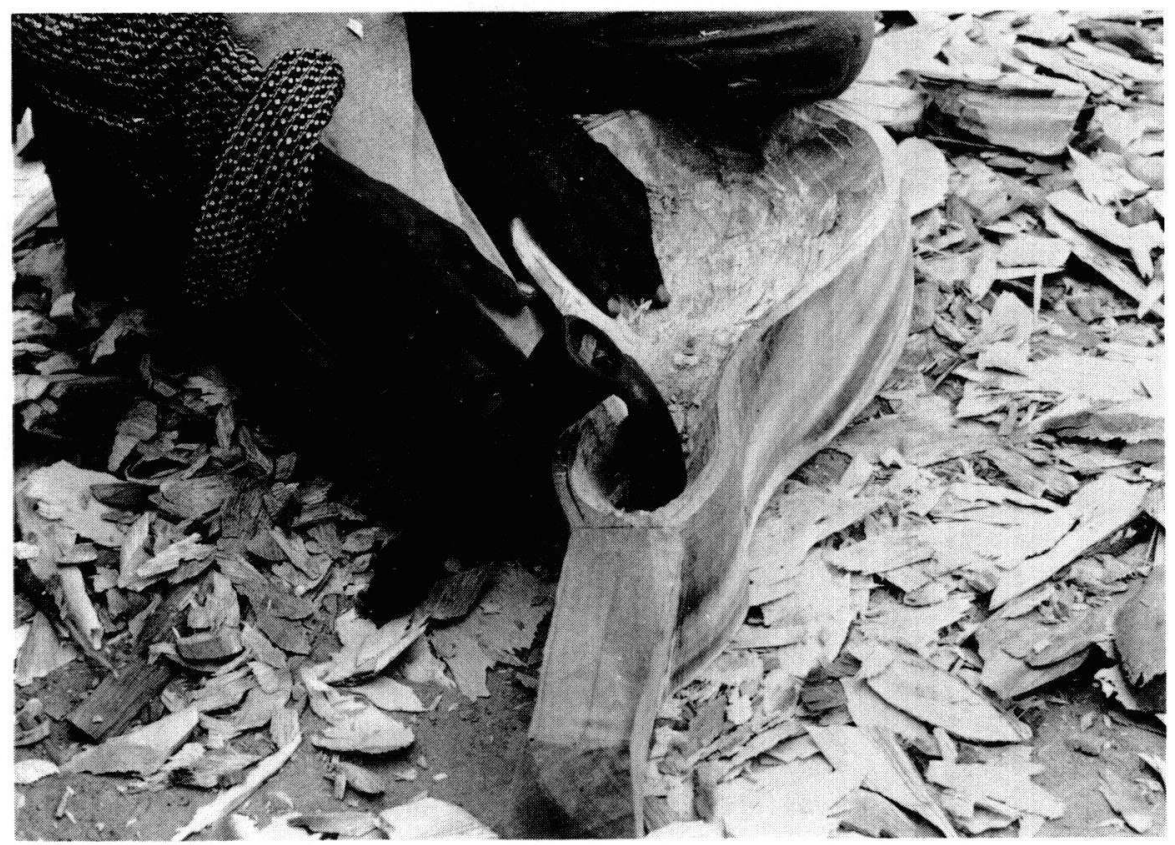

Fig. 3: Outil employé pour le dégrossisage de la cavité de la viola. Photo: L. Fernandez, 1987.



Fig. 4: Emploi du ciseau de menuisier pour le façonnage de l'intérieur de la viola. Photo: L. Fernandez, 1987. 


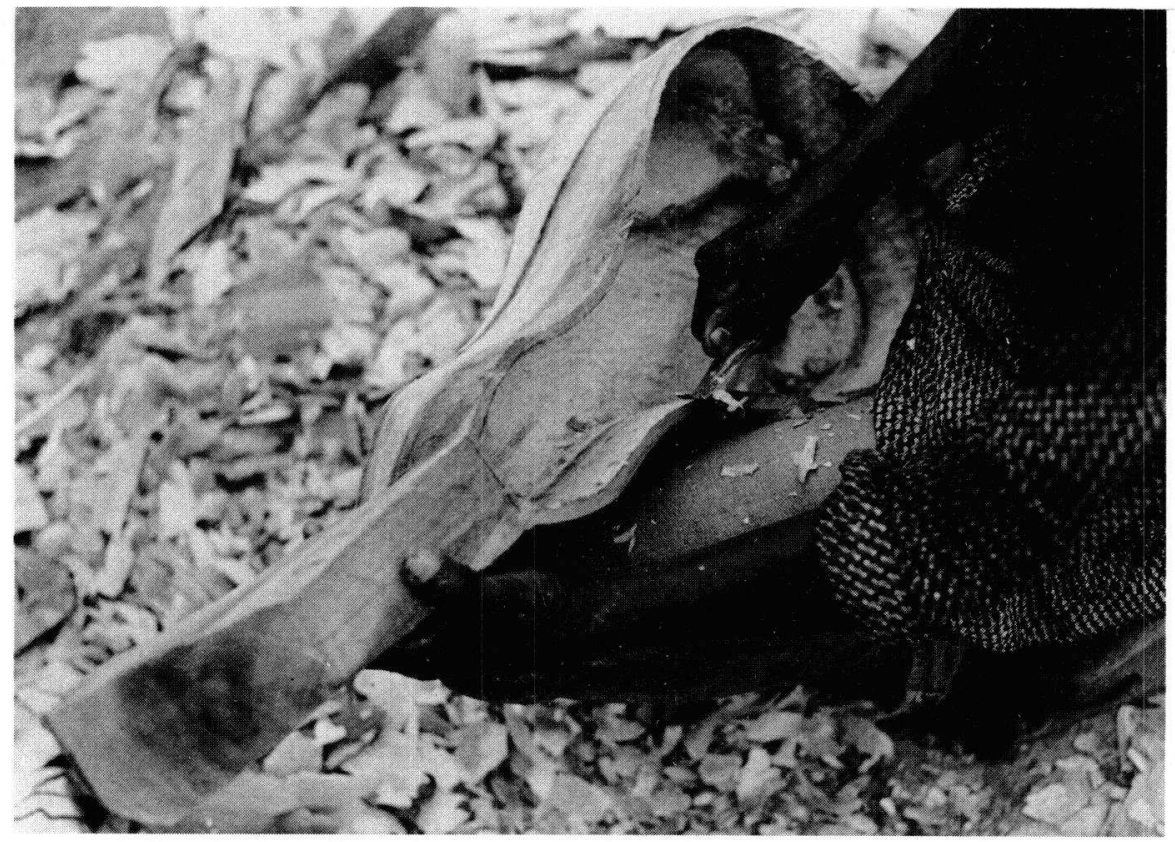

Fig. 5: Un des outils caractéristiques pour la fabrication de la viola de cocho (finition intérieure). Photo: L. Fernandez, 1987.

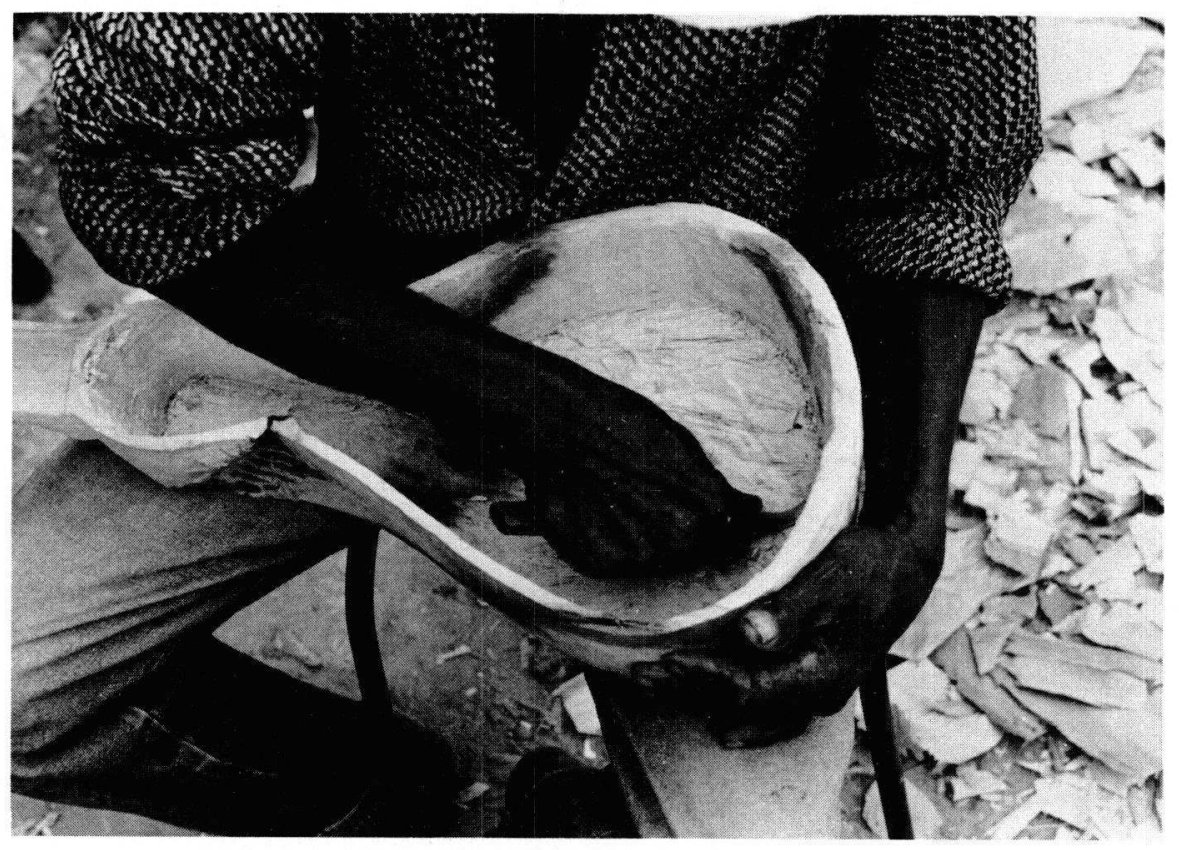

Fig. 6: Finition de la cavité de la viola. Photo: L. Fernandez, 1987. 
directement sur le feu; dans ce cas, il faut agir vite, car cette colle — «violente» selon les informateurs - prend en quelques minutes, En l'absence de sumaré, on a parfois recours à de la colle animale tirée de la vessie natatoire du piranha. Celle-ci doit être «cuite» à la braise, emballeé dans une feuille de bananier. Lorsque la feuille est noire, la colle est prête. Comme en témoigne un des exemplaires de ma collection, plus d'un artisan utilise aujourd'hui une colle industrielle.

Les chevilles, le chevalet et le sillet sont en cèdre. La tête du manche et le diapason sont recouverts d'un placage du même bois, collé par le même procédé que la table. L'artisan «identifie» ses violas par la forme du haut de la tête et par la ligne de séparation entre le diapason et la table d'harmonie. Ces deux détails diffèrent d'un artisan à l'autre, permettant aux initiés de les reconnaître. Les cinq trous recevant les chevilles sont en principe pyrocreusés et légèrement coniques. Les chevilles, droites et postérieures, sont simplement façonnées et fendues au couteau.

La foudre comporte deux ou trois cases délimitées par des ligatures en fil de coton enduites de cire et nouées autour du manche, faisant office de frettes (fig. 7).

Finalement, la viola reçoit deux perforations dans sa partie la plus large, à l'angle entre le bord et le fond de l'instrument, par où est passée une sangle faite avec un simple cordon, qui repose sur l'épaule droite du cururueiro et maintient la viola en position correcte. Lorsqu'on n'en joue plus, on la repousse en arrière sur le dos, ce qui laisse les mains libres au joueur et le fait ressembler à un troubadour du Moyen Age.

A l'exception d'une guirlande multicolore ajoutée quelquefois pour les fêtes, la viola de cochon 'a d'autre finition que le bois brut poncé mais sans ornements.

La table d'harmonie est le plus souvent fermée. Il y a vingt ans, un orifice acoustique d'un centimètre de diamètre était toujours percé près du manche. Mais les artisans ont remarqué qu'il était superflu au moment où la table d'harmonie était bonne. Ainsi recherche-t-on aujourd'hui le son plus doux, plus "velouté», des tables fermées qui ont par ailleurs l'avantage d'empêcher qu'un insecte n'élise domicile à l'intérieur de la viola. Si jamais l'instrument ne «sonne» pas, l'artisan adopte, à son goût, cet orifice pyrocreusé mesurant huit à dix millimètres.

Anciennement, les cordes de la viola étaient en boyau (coati, hérisson, singe, serpent, animaux domestiques). On raconte que les cordes faites des boyaux du serpent, séchés et étirés, «brûlaient» les doigts au bout d'un moment. Quant aux cordes de boyau de chat, elles «sonnent» bien, mais elles sont réputées de mauvaise augure: le cururù finissait presque toujours en bagarre. De nos jours, le nylon (fil de pêche) et la soie filée se sont imposés.

Voici les noms des cordes dans la terminologie du cururù, allant de l'aigu au grave: primera, segunda ou contra, do meio, canotio et de cima. Quelquefois, une sixième corde, la requinta da do meio, est ajoutée en complément à la troisième; elle est accordée une octave plus haut que cette dernière. 


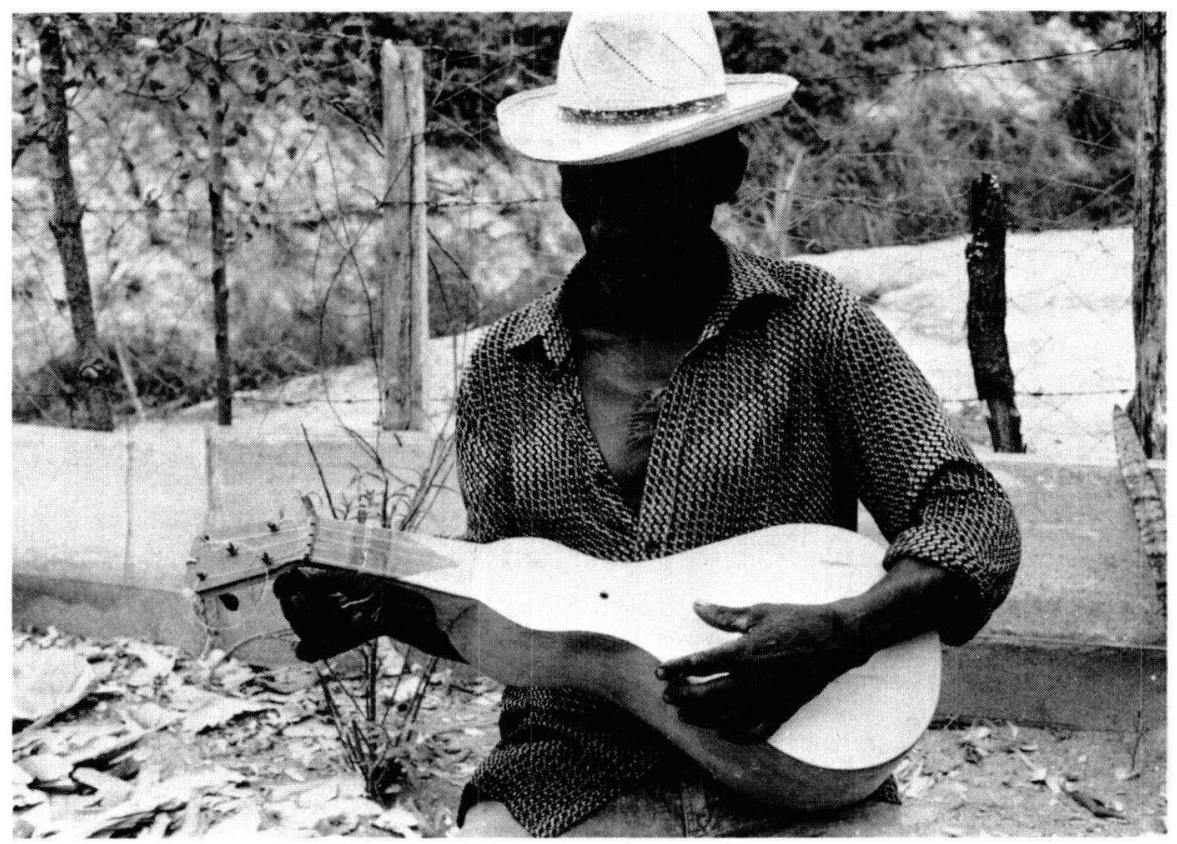

Fig. 7: Manuel Severino Morais, 56 ans, artisan et cururueiro qui fabrique des viola de cocho depuis l'âge de dix ans. Photo: L. Fernandez, 1987.

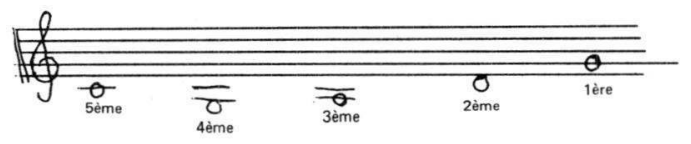

La quatrième corde est très importante, car c'est elle qui «donne le ton». Elle est le plus souvent en soie filée (comme la quatrième de la guitare classique). Autrefois, elle était accordée plus bas ( $\mathrm{sol} b$ ou $f a$ ) pour être employée toujours maintenue (presa) par un doigt sur la première case. Actuellement, elle est accordée en $\mathrm{sol}$ à vide (solta). Les ligatures de coton délimitent des intervalles d'un ton et d'un demi-ton, s'il n'y a que deux cases (cas le plus fréquent), ou de trois fois un demi-ton, si une troisième ligature est ajoutée. L'ambitus déterminé par ces ligatures est d'une octave plus une tierce mineure:

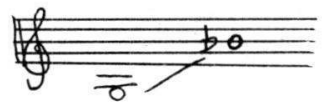

En réalité, lorsque les joueurs s'arnusent à faire du ponteado ${ }^{7}$, la première corde peut être pressée au-delà de la ligature sur tout le manche jusqu'à la ceinture de l'instrument. L'ambitus peut ainsi atteindre deux octaves:

7 De pontear, littéralement «placer les doigts sur les «ponts» (= cases)»; créer une mélodie, par opposition à l'accompagnement avec les accords. 


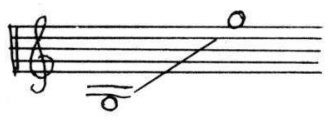

La viola de cocho est toujours jouée en majeur. On emploie l'accord de tonique:

et celui de dominante:
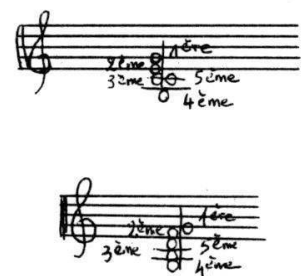

plus parfois un accord «de réponse», de transition:

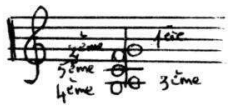

La hauteur de l'accord de la viola de cocho n'est pas fixe. Elle a même souvent tendance à se modifier en cours de jeu. La tonalité indiquée comme étant do peut ainsi passer à ré, voire à ré\# durant une même soirée. A part l'accordage de base (afinação de cocho) les cururueiros en utilisent parfois qui imitent celui d'autres instruments: afinação guitarra ou afinação violãno. Les notes obtenues ne correspondent pas nécessairement à l'échelle tempérée. Les frettes étant mobiles, on s'efforce de les disposer avec précision, de façon à obtenir des intervalles parfois plus petits qu'un demi-ton, mais néanmoins considérés comme «corrects» par l'exécutant. Ce fait doit être pris au sérieux et non pas tenu pour une imprécision ou un manque d'habileté.

\section{Contexte de jeu et accompagnement}

Le cururù $^{8}$ est la danse la plus caractéristique du répertoire de la viola de cocho. Selon plusieurs auteurs, elle aurait été introduite par les Jésuites et serait la plus ancienne, la plus «brésilienne» des danses populaires. Il s'agit d'une danse en cercle exclusivement masculine, aussi appelée função ou brincadeira.

Les fêtes servant de contexte de jeu à la viola de cocho étant généralement dédiées à un saint, les vers chantés commencent par des louanges à celui-ci; ils sont dits de ciência ou de sabeduria. Dans ce cas, les chanteurs sont considérés de primeira («de première catégorie»). Les autres thèmes abordés sont ceux de l'amour, de la nature, de la «femme ingrate», enfin divers thèmes pouvant toucher à la politique ou à l'actualité. Dans ce dernier cas, le cururù permet d'exprimer des idées qui passeraient difficilement ailleurs.

La carreira («rime») est annoncée dans le premier vers. Elle doit être respectée dans l'improvisation qui suit. Voici un exemple de carreira en -ão («São João»):

8 Terme d'origine tupi, qui désigne originellement une variété de crapaud. La danse a-t-elle été appelée ainsi par allusion aux sauts du crapaud? 
«Vou dizé o meu verso

Ne carreira de São João

matei o meu carneiro

prà comé co' meu irmão

Depois do carneiro morto

Quero meu carneiro são

Carreira que tõ cantando

Carreira de São João [...]».

Le seul instrument accompagnant la viola de cocho dans le cururù est le ganz $\grave{a}^{9}$, un idiophone par raclement, fait d'un tube de bambou fendu longitudinalement et strié ou limé transversalement. Il est raclé avec un os ou un autre objet dur.

Les cururueiros, en nombre variable, dansent en tournant dans le sens des aiguilles d'une montre. A tour de rôle, chacun peut sortir du cercle et exécuter un sapateado ou tout autre pas typique du cururù. Le rythme est binaire, moins vif pendant le chant que durant les parties instrumentales. Un rallentando annonce la reprise du chant.

Lorsqu'elles sont grattées avec force, les violas de cocho émettent un crissement provoqué par le frottement des ongles sur les cordes, ce qui leur confère un timbre particulier. Il faut un certain temps au non-initié pour saisir les paroles chantées avec une voix de tête et nasillarde, couverte par les ganzàs et les violas de cocho. La cachaça (alcool de canne) coule à flots, contribuant à maintenir l'inspiration des cururueiros. Il n'est pas rare de les voir passer des nuits entières et prolonger leur prestation le lendemain matin, comme mus par une énergie inépuisable.

Les informateurs sont formels: le cururù, qui ne se pratique aujourd'hui qu'à Cuiabà, n'a rien à voir avec l'ancien cururù rural de São Paulo. Il existe peu de documentation sur les anciens instruments utilisés pour cette danse dans la région de São Paulo; cependant, certaines analogies entre celle-ci et le cururù de Cuiabà sont certainement plus que de simples coïncidences.

Le siriri est une suite de danses, un divertissement ou, comme l'a exprimé un participant, «un jeu pour amuser les Brésiliens». C'est l'adaptation, propre au Mato Grosso, du fandango du littoral de São Paulo, mêlé à d'autres danses, telles la batuque do rio Tietê, la quadrilla, la tirana ou la cirandinha. Il s'agirait d'une synthèse du folklore brésilien, déjà répandue dans une grande partie du Brésil mais actuellement caractéristique du Mato Grosso. Certains auteurs voient l'origine du siriri dans la seguidilla espagnole, appelée siguirilla par les Gitans et dansée également au Portugal, tout comme le fandango. Ils en concluent qu'il s'agit d'une suite de danses d'expression hispano-lusitanienne, mêlée d'éléments africains. J'ai pu observer son expression actuelle au village

9 De $n g a$ 'zà, en dialecte kimbundù d'Angola, ganzà désigne aussi, dans la musique afrobrésilienne, les idiophones de type reco-reco et chocalho en fer blanc. 
de Livramento, près de Cuiabà, où se déroulaient les répétitions précédant la fête de São Benedito. Le rythme était uniquement donné par deux participants qui frappaient chacun à l'aide d'une paire de bâtons sur un rouleau de cuir desseché posé à même le sol. Les violas de cocho, qui d'ordinaire accompagnent cette danse, étaient absentes.

La dança de São Gonçalo est issue des festivités de commémoration de ce saint, le 10 janvier. C'est une danse religieuse. Le saint est représenté avec un instrument à cordes dans les bras, parce qu'il avait la réputation d'animer des danses avec ses fidèles au XIII ${ }^{\mathrm{e}}$ siècle, au Portugal. São Gonçalo est donc le patron des violeiros.

Il s'agit d'une danse en files parallèles, l'une formée d'hommes et l'autre de femmes. A la tête des deux files se trouvent, en nombre variable, les joueurs de viola de cocho et de ganzà. Les vers sont dédiés au saint.

\section{Analyse historique et conclusion}

La viola de cocho est un luth à manche court et à frettes mobiles; ses cordes sont en boyau ou en nylon; sa crosse est fortement inclinée, et ses chevilles sont droites et postérieures. C'est un instrument fait d'une seule pièce de bois évidée, munie d'une table d'harmonie fermée. Certains de ces éléments se retrouvent dans quelques cordophones espagnols archaïques. Selon Julieta de Andrade, on peut supposer que la viola de cocho est dérivée des luths à manche court européens.

Au-delà du manque de documents, déjà évoqué, la profusion de cordophones en Asie et en Europe au Moyen Age et à la Renaissance, ainsi que la confusion taxonomique font que l'origine de l'instrument demeure aléatoire et sujette à spéculation.

Karl von den Steinen (1940), qui se livra à une brève description des fêtes religieuses de Cuiabà lors de son voyage en 1887, parle en ces termes d'un instrument qui accompaganit le cururù: «Koschó, violon à cordes en boyau, que les habitants eux-mêmes fabriquent en bois de salgueiro».

Gabriel Pinto de Arruda, en racontant son séjour à São Luiz de Càceres dans les années trente, précise que le cururù est une danse «exécutée au son d'une ou de plusieurs violas rudimentaires et d'un ou de plusieurs cracachàs ou ganzàs», puis il ajoute:

«[...] la viola avec les divisions du manche réparties de façon originale, a des cordes en boyau d'hérisson, de coati ou de singe [...] une fois les violas ornées de bandes multicolores et bien accordées entre elles, la danse débute [...]» (cité in Andrade 1981: 66).

Un autre auteur, Alceu Maynard de Araujo, décrivait en 1947 un instrument appelé cocho et utilisé dans un cercle de cururù. Il était accompagné d'autres instruments, dont une viola caipira aux cordes métalliques, mais qui 
n'avait pas grand'chose à voir avec la viola de cocho: sa dimension était différente, et elle était monocorde:

"La caisse de résonance, creusée dans le bois, est avec le manche d'une seule pièce [...] sur la partie antérieure de la caisse est fixée une table d'harmonie» (cité in Travassos 1988: 17).

Il existait aussi à São Paulo un certain cocho à quatre cordes:

«Nous connaissons un autre type de cocho, fait comme le précédent, mais qui comporte quatre cordes. Les cordes sont en boyau de singe» (Araujo cité in Travassos 1988: 17).

Selon le même auteur. le cururù se serait diffusé de São Paulo vers l'ouest, en accompagnant les expéditions des bandeirantes:

"Au temps des expéditions et bandeiras ${ }^{10}$, les bandeirantes qui partaient de Piratininga en descendant le Rio Anhembi, dansaient le cururù à l'arrêt le soir; de cette façon, la danse initialement apprise avec les Jésuites s'est propagée dans les régions de pénération des bandeiras» (Araujo 1973: 66).

Toutefois, les cururueiros de Cuiabà maintiennent que le centre de diffusion de la viola de cocho est la capitale du Mato Grosso. Lénine C. Póvoas relève, d'une façon très intéressante, l'influence importante du Rio de la Plata au Mato Grosso:

«[...] des trois fleuves les plus importants du bassin de la Plata, le Paranà, le Paraguay et l'Uruguay, les deux premiers naissent en territoire ayant appartenu à l'ancienne capitainerie de Mato Grosso [...] pour cela et parce qu'ils étaient d'importantes voies navigables par le passé, surtout le rio Paraguay, le Mato Grosso a toujours été intimement lié aux pays de la Plata, en recevant d'eux une grande influence [...] la première expédition d'hommes blancs qui ait atteint ces régions, vint du Rio de la Plata, découvert par Juan Diaz de Solis, en 1515 [...] toutes ces villes vivaient de la navigation fluviale qui venait de Montevideo et Buenos Aires, aux portes de l'Atlantique, jusqu'à Cuiabà, le point le plus lointain dans l'ouest brésilien» (1982a: 9-13).

10 «Expéditions armées qui, partant de la capitainerie de São Vincente (São Paulo par la suite), conquéraient l'intérieur des terres pour capturer des Indiens comme main-d'œuvre et pour rechercher de l'or, de la fin du XVIe au début du XVIII siècle" (In: Médio dicionàrio Aurelio, Nova Fronteira, 1980). 
Pendant de longues années, les liaisons avec Asunción, Buenos Aires et Montevideo étaient beaucoup plus faciles qu'avec Rio de Janeiro: il était courant d'envoyer les jeunes pour être éduqués à Montevideo ou Buenos Aires plutôt qu'à Rio de Janeiro ou São Paulo. Parmi les émigrants qui tentaient leur chance au Mato Grosso, il y avait beaucoup d'Européens, notamment des Italiens et des Espagnols, qui apportaient avec eux les coutumes des pays de la Plata, qu'ils avaient assimilées au cours de séjours précédant leur voyage.

Il n'est donc pas étonnant de trouver une histoire populaire relatant l'origine de la viola de cocho en rapport avec les pays de la Plata. Voici le récit que nous avons recueilli en 1987 auprès de Luiz Marques da Silva, ancien cururueiro et ex-président de l'Association de folklore du Mato Grosso:

«Lorsque Cuiabà était encore une petite bourgade, vivait au bord de la rivière un artisan qui faisait des pirogues, des gamelles, des cuillers en bois, des auges, des mangeoires et d'autres ustensiles dans le même matériau. Un voyageur, peut-être un Paraguayen, apparut un jour en remontant la rivière. Il transportait avec lui un instrument à cordes qui pouvait être une viola ou une guitare. Il fut hébergé quelque temps par l'artisan qui apprit un peu à jouer de cet instrument. Lorsque le voyageur repartit — n'ayant pas trouvé de travail - il emporta avec lui son instrument. De là surgit chez l'artisan l'idée d'en fabriquer un exemplaire. L'artisan trouva plus naturel et plus facile de faire l'instrument d'un seul bloc de bois, en ajoutant uniquement la table d'harmonie en fin de travail. Lorsqu'il présenta l'instrument, il fut questionné: 'Qu'est-ce que c'est? - Une viola. - Une viola de quoi? - Une viola de cocho (par allusion aux mangeoires qu'il construisait et par plaisanterie). C'est comme ça que le nom lui est resté».

Quelle qu'en soit l'origine, voilà cet instrument implanté dans la tradition de Cuiabà depuis deux siècles ou deux siècles et demi. Certains artistes, comme Guapo à Cuiabà, Fernando Corrêa à Brasilia et d'autres, s'efforcent de le promouvoir en lui donnant une place dans les spectacles de musique traditionnelle et populaire hors des frontières du cururù et de Cuiabà.

Dans le monde musical brésilien "transistorisé» et "américanisé» à souhait, la viola de cocho parviendra-t-elle à se maintenir?

\section{Bibliographie}

ANDRADE Julieta de

1977 «Pesquisa de folclore no Mato Grosso». Revista Cultura (São Paulo) 25: 89-96.

1981 Cocho Matogrossense: um alaùde brasileiro. São Paulo: Escola de Folclore (thèse inédite).

JENKINS Jean

1983 Man and Music. A Survey of Traditional Non-European Musical Instruments. Edinburgh: Royal Scottish Museum. 
MELLO Maria Lùcia de

1982 «A Dança de São Gonçalo». In: Centro Letras-Ciências humanas, Dep. Artes (Coleção pesquisas $\mathrm{N}^{\circ} 1$ ), p. 41-52.

PIRES DE ALMEIDA Benedicto

1939 "A festa do Divino. Tradicões e reminiscências de Tietê». Revista do Arquivo Municipal (São Paulo) LIX.

PÓVOAS Lénine $C$.

1982a Influências do Rio da Prata em Mato Grosso (ensaio). Cuiabà: Éd. par l'auteur.

1982b Historia da Cultura Matogrossense. Cuiabà: Éd. par l'auteur.

RAMOS Otàvio \& DRUMOND Arnaldo F.

1979 «Centro de Cultura da região Centro-Oeste». Boletim cultural (Cuiabà) 2: 21-26.

TRAVASSOS Elizabeth \& NUNES CORRÔEA Roberto

1988 Viola de Cocho. Brasilia/Rio de Janeiro: Ministerio da Cultura/Fundação Nacional de Arte (Instituto Nacional do folclore).

VON DEN STEINEN Karl

1940 Entre os Aborigines do Brasil Central. São Paulo: Departemento de Cultura (p. 34-58). 